\title{
Rheological Aspects of the Intermolecular Interaction between Xanthan and Locust Bean Gum in Aqueous Media
}

\author{
Masakuni Tako, Atsushi Asato and Sanehisa NaKamura \\ Department of Agricultural Chemistry, Faculty of Agriculture, \\ University of the Ryukyus, Nishihara-cho, Okinawa 903-01, Japan
}

Received April 20, 1984

\begin{abstract}
Non-Newtonian behavior and dynamic viscoelasticity of a series of aqueous mixed solutions of xanthan and locust bean gum were measured using a rheogoniometer, and the rheological properties were analysed. A gelation occurred in the mixture at the concentration of $0.2 \%$ total gums at room temperature. The flow curves of the mixture solutions showed a yield value and approximated to plastic behavior at $50^{\circ} \mathrm{C}$. The maximum dynamic modulus was obtained when the mixing ratio of xanthan to locust bean gum was $1: 2$, while comparable high moduli were also obtained in the mixing ratio of $1: 3$ or $1: 4$. A mixture of deacetylated xanthan and locust bean gum showed the highest dynamic modulus, about two times that of the mixture of native or Na-form xanthan. The dynamic modulus of the mixtures decreased rapidly with increasing temperature. In contrast, the dynamic viscosity was scarcely changed during increasing temperature in the mixing ratio of $2: 1$. The dynamic modulus was decreased by addition of urea $(4.0 \mathrm{M}), \mathrm{NaCl}(0.1 \%)$ and $\mathrm{MgCl}_{2}$. We concluded that the intermolecular interaction between xanthan and locust bean gum might occur between the side chains of the former and backbone of the latter, as in a lock-and-key effect.
\end{abstract}

Xanthan is an extensively investigated polysaccharide bacause of its unique rheological properties. ${ }^{1 \sim 3)}$ We have proposed that xanthan is associated in quaternary between charged trisaccharide side chains ${ }^{2}$ and the acetyl residue contributes its intramolecular association. ${ }^{4}$ It has also been suggested that the tertiary structure gives a 5-fold single stranded helix by an X-ray scattering study of the solid state. $\left.{ }^{5}\right)$ On the other hand, the synergistic interaction between xanthan and locust bean gum in aqueous solution is well known. ${ }^{6,7)}$ Dea et $a l^{8)}$ have reported that the synergistic interaction between xanthan and galactomannan is closely correlated with the degree of substitution of the mannan chain. Guar gum, in which the ratio of mannose to galactose is close to $2: 1$, does not gel with xanthan at any concentration. Soft, fairly weak gels are obtained with tara gum, where the mannose to galactose ratio is around $3: 1$, as against $4: 1$ in locust bean gum, which gives far stronger and more rigid gels. Accordingly, Dea et al. ${ }^{8)}$ have proposed a mechanism for the interaction, though the function of the side chains of xanthan is neglected.

We confirmed that the association characteristics of the gums depend on the charged trisaccharide side chains of xanthan. ${ }^{2,4)}$ Thus, we analyze here the rheological behavior of a mixture of xanthan and locust bean gum so as to propose a mechanism of the molecular interaction in aqueous solution.

\section{MATERIALS AND METHODS}

Purification of polysaccharides. Xanthan and locust bean gum identical to those in previous studies ${ }^{2,4,9)}$ were obtained from Taiyo Kagaku Co., Ltd. Xanthan was purified by the same manner as previously reported. ${ }^{4}$ Locust bean gum was dissolved in hot water $\left(85^{\circ} \mathrm{C}\right)$ at $0.5^{\circ}$ and filtered 
through a Celite 545 layer. Then a double volume of ethanol was added, polysaccharide was precipitated, and it was dried in vacuum.

Conversion into Na-form. Purified xanthan was dissolved in water again, and deionized by passing through a column of Amberlite IR $120\left(\mathrm{H}^{+}\right)$, then neutralized with $0.05 \mathrm{~N} \mathrm{NaOH}$. In the presence of $0.5 \% \mathrm{NaCl}$, a double volume of ethanol was added, and polysaccharide was precipitated and was dried in vacuum.

Deacetylation of xanthan. The deacetylated xanthan was prepared in the same manner reported previously. ${ }^{4)}$ The acetyl content was $4.6 \%$. Recovery was $95 \%$ of the theoretical maximum.

Preparation of mixed solutions of xanthan and locust bean gum. To make various solutions of xanthan and locust bean gum, with the total gum concentration constant at $0.2 \%$, locust bean gum was dissolved in hot water $\left(85^{\circ} \mathrm{C}\right)$, then xanthan was dissolved in addition, unless stated otherwise.

Liquid chromatography. Locust bean gum $(50 \mathrm{mg})$ was dissolved in $25 \mathrm{ml}$ of $2 \mathrm{~N}$ hydrochloric acid and heated for $3 \mathrm{hr}$ at $100^{\circ} \mathrm{C}$. After cooling in an ice bath, the hydrolyzate was neutralized with silver carbonate and filtered through Celite 545 to remove silver chloride. Excess silver ions were precipitated with hydrogen sulfide, and the solution was reduced in volume and filtered through Celite 545 into a $10 \mathrm{ml}$ volumetric flask. High performance liquid chromatography was performed with a Shimadzu LC-4A chromatograph using a column of ISA-07/S2504 and a mobile phase of $2.0 \mathrm{M}$ boric acid adjusted to $\mathrm{pH} 8.5$ with $0.1 \mathrm{~N} \mathrm{KOH}$. The reaction conditions were as follows: $1.0 \%$ arginine and $3.0 \%$ boric acid; temperature, $150{ }^{\circ} \mathrm{C}$; flow rate, $0.5 \mathrm{~cm} / \mathrm{min}$.

Measurement of specific rotation. Specific rotation was measured at $589 \mathrm{~nm}$ on an automatic digital polarimeter DIP-180 (Japan spectroscopic Co., Ltd.) at $0.5 \%(\mathrm{w} / \mathrm{v})$ in water.

Determination of molecular weight. The molecular weight of locust bean gum was determined by the viscometric method following the relationship, ${ }^{10)}[\eta]=3.8 \times$ $10^{-4} \cdot M_{w}{ }^{0.723}$. Intrinsic viscosity, $[\eta]$, was detemined for each solution ranging from $0.05 \sim 0.3 \%$ by measuring the specific viscosities with Ostwald type viscometer at $25^{\circ} \mathrm{C}$. The flow rate for water was $44 \mathrm{sec}$.

Viscosity and dynamic viscoelasticity measurements. Viscosity at different shear rates $\left(1.667 \sim 66.685 \mathrm{sec}^{-1}\right)$ and dynamic viscoelasticity at steady angular velocity $(3.768$ $\mathrm{rad} / \mathrm{sec}$ ) measurements were performed on a rheogoniometer, a coaxial cylinder with a rotating outer cylinder, dimensions: length, $6.0 \mathrm{~cm}$; outer cylinder, $2.2 \mathrm{~cm}$ in diameter; inner cylinder, $1.6 \mathrm{~cm}$ in diameter (IR-103, Iwamoto Seisakusho Co., Ltd.).

The temperature of the sample was controlled by circulating oil from a thermo-cool (LCH-130F, Toyo, Co., Ltd.) over the temperature range of $20 \sim 60^{\circ} \mathrm{C}$ and raised at a rate of $1{ }^{\circ} \mathrm{C}$ a minute by steps.

Shear rate, $D$, shear stress, $S$, and apparent viscosity, $\eta$, were calculated with the equation of Margules. ${ }^{11)}$ Dynamic viscosity, $\eta^{\prime}$, and elasticity, $G^{\prime}$, were calculated by a modification of Markovitz's equation. ${ }^{12)}$

\section{RESULTS AND DISCUSSION}

As the intermolecular interaction between xanthan and galactomannan is closely correlated with the degree of substitution of the mannan chain, as mentioned above, the degree of substitution of locust bean gum was determined by liquid chromatography as shown in Fig. 1, and calculated to be D-mannose: Dgalactose $=4.4: 1.0$. The molecular weight of locust bean gum was estimated to be 263,000 . The specific rotation, $[\alpha]_{589}^{20}$, was $+22^{\circ}$ at $0.5 \%$ in water.

Although neither xanthan nor locust bean gum gelled alone, a mixture of the gums gave a gel at $0.2 \%$ total gums at room temperature. Flow curves of a mixture of native (Fig. 2(A))and deacetylated (Fig. 2(B)) xanthan with locust bean gum solutions at the ratio of the two gums are altered, keeping the total concentration constant at $0.2 \%$ at $50^{\circ} \mathrm{C}$, is shown in Fig. 2. All of the mixed solutions were shifted over to the left from that of xanthan alone.

Figure 3 shows the effect of the combination ratio of xanthan (native, Na-form, and deacetylated)-locust bean gum solution on dynamic viscoelasticity at $0.2 \%$ total gums at $25^{\circ} \mathrm{C}$. The maximum gelation was achieved when the mixing ratio of xanthan to locust bean gum was $1: 2$, while comparably strong gels were also obtained with ratios of $1: 3$ and $1: 4$. In the case of deacetylated xanthan, a much stronger gel was observed, about twice as strong as the others, indicating that much more intense intermolecular interactions were developed by the deacetylation of residues on the side chains of inner mannose residues at O-6. The results suggest that there are about 
(a)

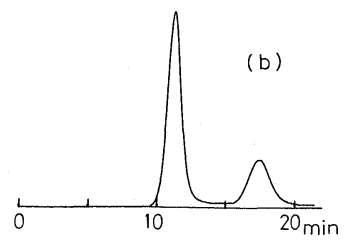

Fig. 1. Liquid Chromatogram of Hydrolyzate of Locust Bean Gum.

Column, ISA-07/S2504; mobile phase, $2.0 \mathrm{M}$ boric acid

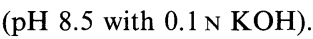

Reaction conditions: reagent, $1.0 \%$ arginine in $3.0 \%$ boric acid; temperature, $150^{\circ} \mathrm{C}$; flow rate, $0.5 \mathrm{ml} / \mathrm{min}$. (a), Dmannose; (b), D-galactose.

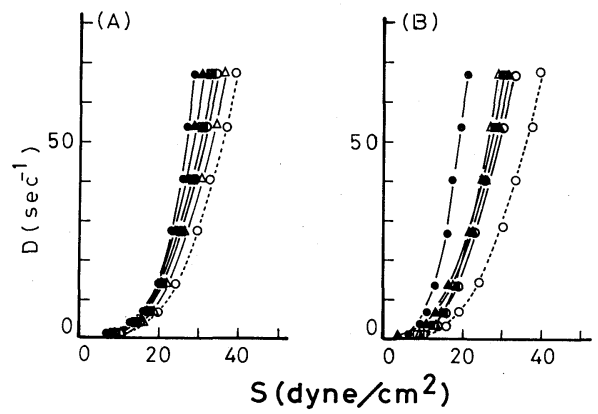

Fig. 2. Flow Curves of Xanthan Solution Mixed with Locust Bean Gum at $0.2 \%$ Total Gum at $50^{\circ} \mathrm{C}$.

Combination ratio of xanthan to locust bean gum: $4: 1 ; \triangle, 2: 1 ; \bigcirc, 1: 1 ; \bigcirc, 1: 2 ; \boldsymbol{\Delta}, 1: 4 ; \_$, mixed solution; --.-, xanthan alone $(0.2 \%)$. (A), native xanthan; (B), deacetylated xanthan.

twice the number of junction sites available on each xanthan molecule as on the locust bean gum, and also suggest that the molecule becomes more flexible and can associate with locust bean gum more easily after deacetylation, probably because they are free from the intramolecular association to which the acetyl residues contribute. ${ }^{4)}$ It was furthermore suggested that the side chains of xanthan may contribute to the interaction with locust bean gum. On the other hand, the dynamic viscosity of the mixed solutions showed remarkable low values.

Figures $4(\mathrm{~A}),(\mathrm{B})$, and (C) show the effect of temperature on the dynamic viscoelasticity of a mixed solution of native, Na-form, and

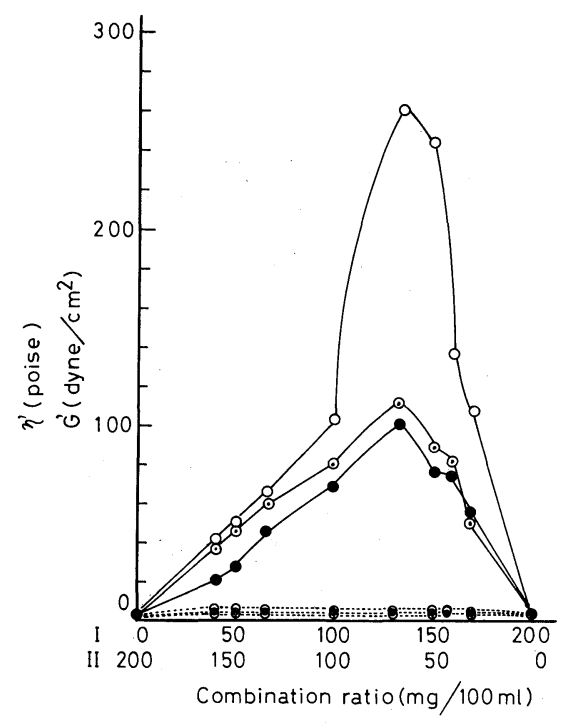

FIG. 3. Dynamic Viscoelasticity of $0.2 \%$ Xanthan/ Locust Bean Gum Solution as a Function of Combination Ratio at Angular Velocity of $3.768 \mathrm{rad} / \mathrm{sec}$ and $25^{\circ} \mathrm{C}$.

, native xanthan; $\odot$, Na-form; $\bigcirc$, deacetylated. I, locust bean gum; II, xanthan.

deacetylated xanthan-locust bean gum. The dynamic modulus showed the highest values at the mixing ratio of $1: 2$ for each mixture. The results agreed with those in Fig. 3. The dynamic modulus decreased rapidly with increasing temperature. On the other, the dynamic viscosity showed very low values in comparison with those of the dynamic modulus. It decreased rapidly with increasing temperature for xanthan to locust bean gum ratio of $1: 2$ to $1: 4$, but scarce changed from $2 ; 1$ to $4: 1$, whose insensitivity agreed with that of xanthan alone. ${ }^{2)}$ Such an insensitivity in dynamic viscosity of xanthan alone resulted from quaternary association $^{2)}$ and the intramolecular association contributed by acetyl residues. ${ }^{4)}$ Thus, the results indicate that some of the non-association sites on the xanthan molecule remained. The results also suggest that the side chains of xanthan may play a role in the interaction.

The dynamic viscoelasticity of a mixed solution of native xanthan and locust bean gum was decreased by the addition of urea (4.0, 6.0, and $8.0 \mathrm{M}$ ), as shown in Fig. 5. This suggests 


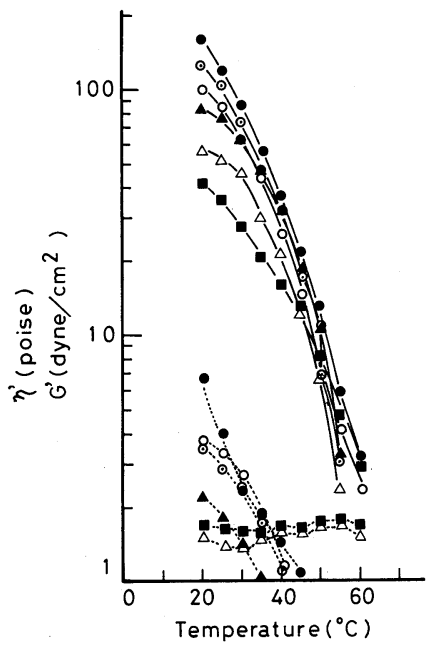

Fig. 4(A). Effects of Temperature on Dynamic Viscoelasticity of Mixed Solutions of Native Xanthan and Locust Bean Gum at $0.2 \%$ Total Gum at Angular Velocity of $3.768 \mathrm{rad} / \mathrm{sec}$.

Combination ratio of xanthan to locust bean gum: $4: 1 ; \triangle, 2: 1 ; \bigcirc, 1: 1 ; \bigcirc, 1: 2 ; \odot, 1: 3 ; \mathbf{\Delta}, 1: 4$.

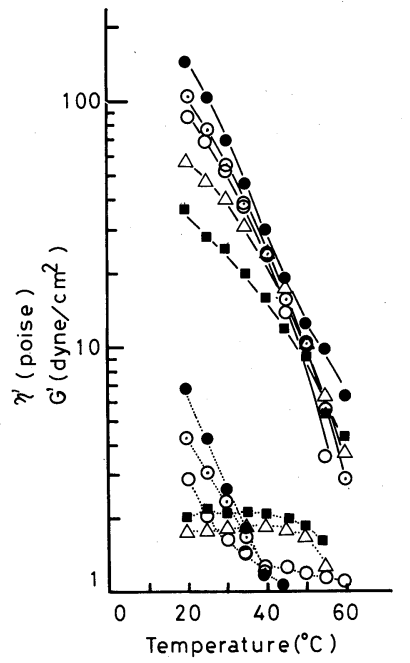

Fig. 4(B). Effects of Temperature on Dynamic Viscoelasticity of Mixed Solutions of Na-form Xanthan and Locust Bean Gum at $0.2 \%$ Total Gum at Angular Velocity of $3.768 \mathrm{rad} / \mathrm{sec}$.

The symbols are the same as in Fig. 4(A).

that hydrogen bonding takes place in the interaction.

In spite of an increase in dynamic viscoelasticity of xanthan alone by addition of salt,${ }^{2)}$ the dynamic modulus of the mixture with locust

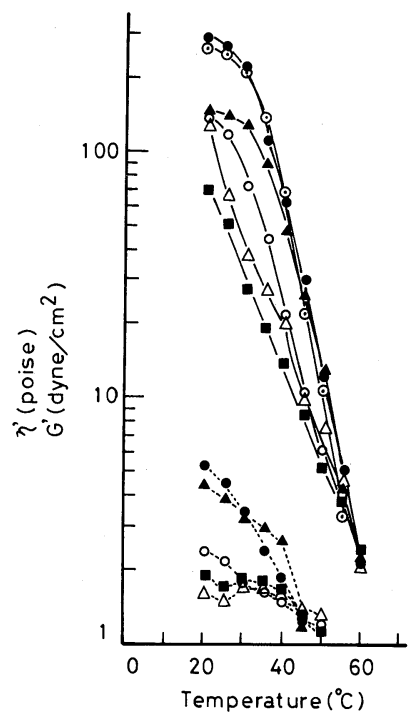

FIG. 4(C). Effects of Temperature on Dynamic Viscoelasticity of Mixed Solutions of Deacetylated Xanthan and Locust Bean Gum at $0.2 \%$ Total Gum at Angular Velocity of $3.768 \mathrm{rad} / \mathrm{sec}$.

The symbols are the same as in Fig. 4(A).

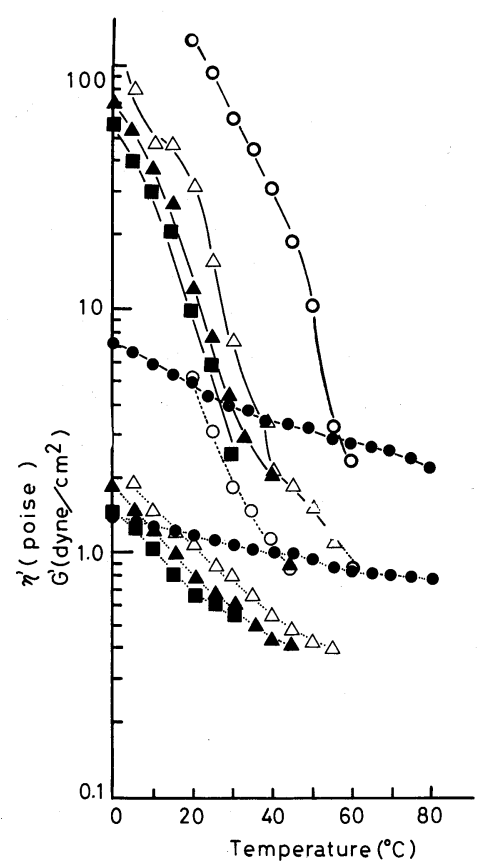

FIG. 5. Effects of Temperature on Dynamic Viscoelasticity of a Mixed Solution of Native Xanthan and Locust Bean Gum with Addition of Urea at Angular Velocity of $3.768 \mathrm{rad} / \mathrm{sec}$.

Xanthan/locust bean gum, $1: 2 \quad(0.2 \%$ total gum). Concentration of urea: $\bigcirc$, no addition; $\triangle, 4.0 \mathrm{M} ; \boldsymbol{\triangle}, 6.0 \mathrm{M}$; 口, $8.0 \mathrm{M}$; $\bigcirc$, xanthan alone $(0.2 \%)$. 


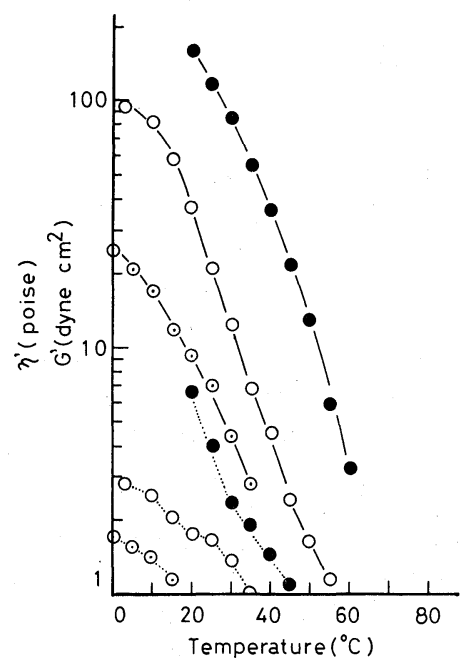

FIG. 6. Effects of Temperature on Dynamic Viscoelasticity of a Mixed Solution of Native Xanthan and Locust Bean Gum with Addition of Salts at Angular Velocity of $3.768 \mathrm{rad} / \mathrm{sec}$.

Xanthan/locust bean gum, $1: 2(0.2 \%$ total gum $)$. $\mathbf{O}$, no addition of salt; $\bigcirc, 0.1 \% \mathrm{NaCl} ; \odot, 0.1 \% \mathrm{MgCl}_{2}$.
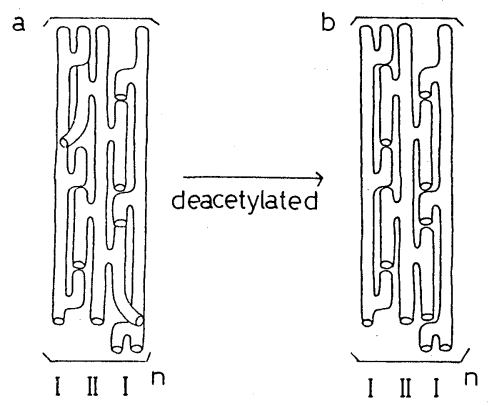

Fig. 7. A Possible Mechanism of the Intermolecular Interaction between Xanthan and Locust Bean Gum in Aqueous Solution.

The models are viewed perpendicular to the axis. A molecule of xanthan can combine with two or more molecules of locust bean gum, as in a lock-and-key effect. As the side chains of xanthan are somewhat rigid because of its intramolecular association between acetyl residues and backbone, incomplete interaction sites may exist partially (a), in which the interaction may be accomplished by the deacetylation of xanthan (b). I, locust bean gum; II, xanthan.

bean gum decreased in the presence of $\mathrm{NaCl}$ or $\mathrm{MgCl}_{2}$ even at $0.1 \%$ as shown in Fig. 6. The dissociation might be brought about ionic repulsion on charged trisaccharide side chains of xanthan due to $\mathrm{Na}^{+}$or $\mathrm{Mg}^{+2}$. The results also suggest that the side chains of xanthan contribute to the interaction.

We conclude that the intermolecular interaction between xanthan and locust bean gum may occur between side chains of the former and the backbone of the latter as illustrated in Fig. 7a. The structural integrity of a polysaccharide gel is maintained by interactions in which the molecules adopt the same ordered conformation as in the solid state. ${ }^{13)}$ Accordingly, as the tertiary structure of xanthan may be a 5-fold single-stranded helix, ${ }^{5)}$ the side chains of xanthan insert into adjacent unsubstituted segments of the backbone of locust bean gum which is extended in a 2-fold ribbon-like structure ${ }^{8,14)}$ aligning parallel or antiparallel, as in a lock-and-key effect. According to this mechanism, a molecular chain of xanthan can associate with two or more backbone of the locust bean gum, in which combination the ratio may be a favorable conformation in aqueous solution. According to the X-ray scattering study, ${ }^{5)} 5 / 1$ or $5 / 2$-fold structure is favorable for xanthan in the solid state. However, the experimental results suggest that $5 / 2$ - or $5 / 3$-fold structure is favorable in aqueous solution, because the maximum interaction is achieved at the mixing ratio of $1: 2$. In the mixture of deacetylated xanthan, very intense interaction is also achieved in the ratio of $1: 3$ and the degree of dynamic modulus is about two times over a wide range of combination ratio. The phenomenon may be brought about by the interaction change. That is, since the side chains of native xanthan are somewhat rigid because of its intramolecular association contributed by acetyl residues, incomplete interaction sites may exist (a), in which the interaction may be accomplished by the deacetylation of xanthan (b). Although it has been reported that the side chains unit of locust bean gum is in uniform blocks along the mannan backbone, ${ }^{15)}$ the mechanism of the interaction is independent of the structure, because each junction site may be accomplished within three sugar residues, including pyruvate and acetate residues of the side chains of xanthan. The 
more intense interaction in the mixture of deacetylated xanthan suggests that a regular branching unit in locust bean gum probably partially exists, as illustrated in Fig. 7. Studies on synergistic interaction between xanthan and guar gum solution will be published elsewhere. ${ }^{16)}$

Acknowledgments. We wish to thank Dr. Akira Misaki, Professor of Osaka City University for his valuable advice. We also thank Mr. Katsushi Tokumine for his technical assistance.

\section{REFERENCES}

1) A. Jeanes, J. E. Pittsley and F. R. Senti, J. Appl. Polym. Sci., 5, 519 (1961).

2) M. Tako, T. Nagahama and D. Nomura, Nippon Nôgeikagaku kaishi, 51, 513 (1977).

3) P. J. Whitcomb and C. W. Macosko, J. Rheology, 22, 493 (1978).
4) M. Tako and S. Nakamura, Agric. Biol. Chem., 48, 2987 (1984).

5) R. Moorhous, M. D. Walkinshaw and S. Arnott, Am. Chem. Soc. Sym. Ser., 45, 90 (1977).

6) J. K. Rocks, Food Technol., 25, 476 (1971).

7) D. A. Rees, Biochem. J., 126, 257 (1972).

8) I. C. M. Dea, E. R. Morris, D. A. Rees, E. J. Welsh, H. A. Barnes and J. Price, Carbohydr. Res., 57, 249 (1977).

9) M. Tako, T. Nagahama and D. Nomura, Nippon Nôgeikagaku Kaishi, 51, 397 (1977).

10) G. Robinson, S. B. R. Murphy and E. R. Morris, Carbohydr. Res., 107, 17 (1982).

11) J. Harris, "Rheology and Non-Newtonian Flow," Longman Inc., New York, 1977, p. 30.

12) H. Markovitz, J. Appl. Phys., 23, 1070 (1952).

13) D. A. Rees, "Advan. Carbohyd. Chem. Biochem.," Academic Press, 1969, 24, p. 303.

14) P. Zugenmaier, Biopolymer, 13, 1127 (1974).

15) C. W. Baker and R. L. Whistler, Carbohydr. Res., 45, 237 (1975).

16) M. Tako and S. Nakamura, Carbohydr. Res., in press. 